\section{Les cellules souches pluripotentes font peau neuve}

Xavier Nissan, Gilles Lemaitre, Marc Peschanski, Christine Baldeschi
Inserm/UEVE UMR-861, ISTEM, AFM,

Institut des cellules souches

pour le traitement et l'étude

des maladies monogéniques,

5 , rue Henri Desbruères,

91030 Évry Cedex, France.

cbaldeschi@istem.fr

l'ontogenèse. Cette prise en compte nous avait permis d'obtenir la différenciation de CSEh en neurones moyens-épineux GABAergiques du striatum [5]. Dans le cas de la peau, les protocoles testés précédemment s'appuyaient sur l'idée qu'une induction des CSEh limitée dans le temps permettrait d'orienter les cellules vers le lignage kératinocytaire; notre démarche au contraire a été de maintenir le traitement par des cytokines et des agents pharmacologiques des cellules jusqu'à l'apparition du phénotype cellulaire recherché. L'engagement des CSEh dans le lignage épidermique a ainsi été rendu possible en combinant à la fois leur coculture en présence de cellules nourricières et un traitement pharmacologique de plus de 40 jours (Figure 1A). Les CSEh ont été traitées avec de l'acide ascorbique et la protéine morphogène de type 4 (BMP4), et placées dans les conditions de cultures adaptées à la survie et à la prolifération de kératinocytes définies par les travaux princeps de Rheinwald et Green [6]. Nous avons suivi l'évolution du profil d'expression génétique des cellules au fur et à mesure du processus de différenciation et montré qu'elle respectait effectivement la chronobiologie du développement embryonnaire de la peau humaine. Dès les premiers jours, l'expression de la protéine SSEA3 diminue, suggérant la perte des capacités pluripotentes des CSEh (Figure 1B). Au jour 10 de culture, parallèlement à l'acquisition d'une morphologie de type épithélial, les cellules expriment transitoirement

la kératine 18, normalement exprimée
Un protocole de différenciation fondé sur la chronobiologie de l'ontogenèse La réussite de notre équipe tient vraisemblablement en premier lieu à l'application d'un concept qui s'impose aujourd'hui progressivement dans toutes ces recherches, celui du respect de la chronobiologie observée durant souches embryonnaires humaines (CSEh)

> Ces deux dernières décennies, les avancés technologiques effectuées dans pronostic vital des grands brûlés. À ce jour, le principal traitement disponible pour ces patients est l'autogreffe d'épiderme. Cette technique consiste à effectuer une biopsie cutanée de quelisoler les progéniteurs kératinocytaires de rejet immunitaire et de transmission d'agents pathogènes.

Grâce à leurs capacités d'autorenouvellement et de pluripotence, les cellules

contenant du collagène bovin et/ou des cellules de peau allogéniques [2]. Cependant, ces technologies ne perpermis d'améliorer considérablement le

représentent une source illimitée de celinduite «à la demande»dans l'ensemble des types cellulaires de l'organisme. La reconstitution d'un épiderme à partir de kératinocytes dérivés de CSEh représenterait donc une alternative thérapeutique à l'utilisation de ces pansements biologiques transitoires. Des études préalables ont montré que les CSEh et leurs dérivés présentaient un faible niveau d'expression d'antigènes majeurs du CMH (complexe majeur d'histocompatibilité), laissant penser que la réponse de l'hôte contre le greffon pouretre limitée ou retardée, permet Plusieurs équipes internationales ont essayé de transposer ce modèle à la dans un premier temps prometteurs, n'avaient cependant pas permis jusde publier un protocole de différenciahomogène de kératinocytes capables de reconstituer un épiderme pluristratifié aussi bien in vitro qu'in vivo [4]. 


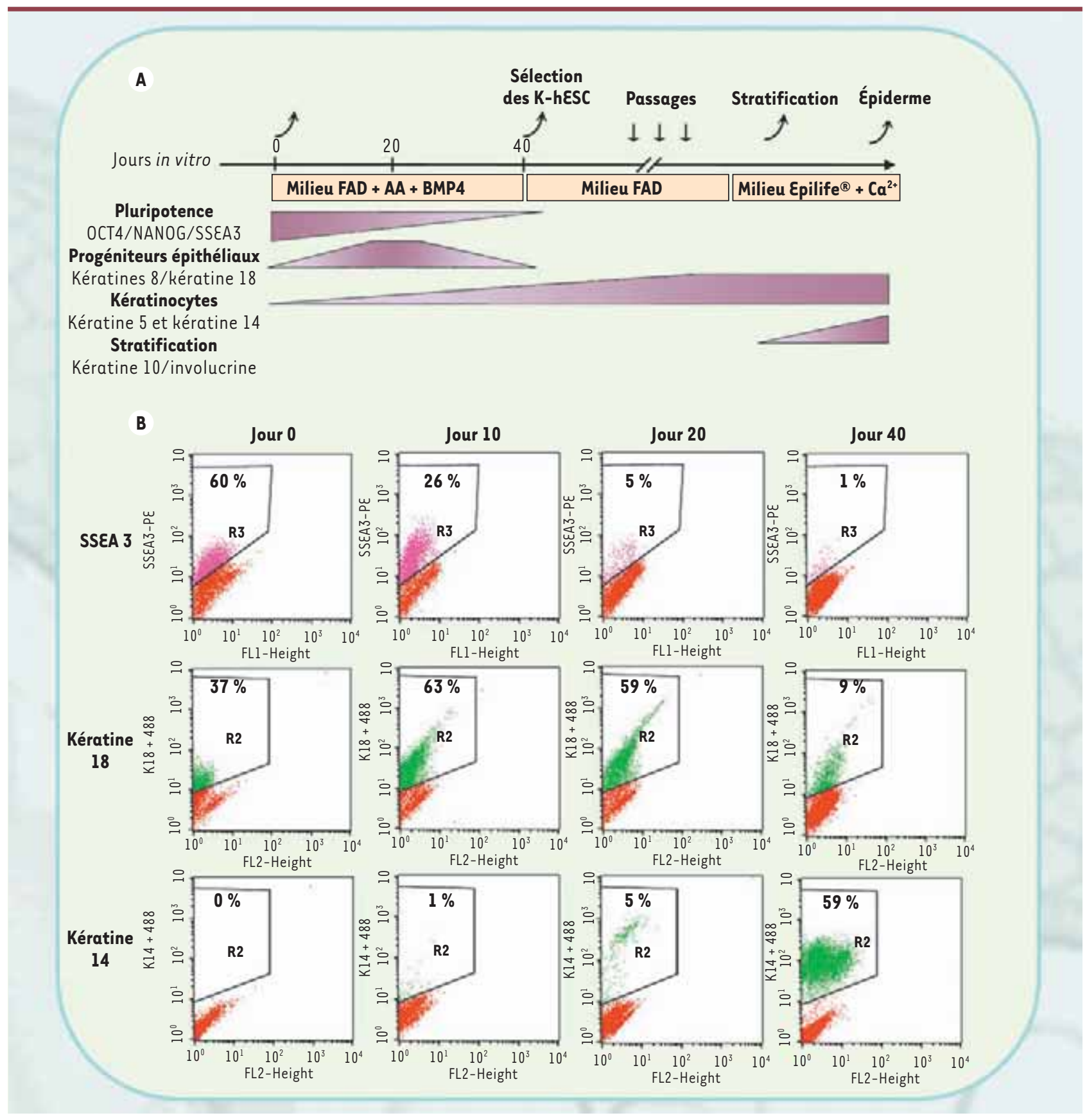

Figure 1. Processus de différenciation des cellules souches embryonnaires humaines en kératinocytes. Les CSEh sont différenciées en kératinocytes après 40 jours de traitement par différents agents pharmacologiques et par des cytokines mimant les signaux impliqués dans la formation de l'épiderme au cours du développement embryonnaire humain. On retrouve l'expression des marqueurs caractéristiques de chacune des étapes du développement ( $A$ ) et une chute rapide des marqueurs de pluripotence (OCT4, NANOG et SSEA3), l'expression transitoire des marqueurs des progéniteurs épithéliaux (kératines 8 et 18), puis l'expression des marqueurs des kératinocytes basaux (kératines 5 et 14) et enfin ceux des couches supérieures de l'épiderme (la kératine 10 et l'involucrine). L'analyse par cytométrie de flux confirme qu'une fois le processus de différenciation enclenché, les CSEh perdent l'expression de SSEA3, expriment transitoirement la kératine 18 puis la kératine 14 (B). AA : acide ascorbique; BMP4 : bone morphogenetic protein 4 ; FAD : milieu de culture fait d'un mélange de milieu Dulbecco et Ham F12.

par les progéniteurs épithéliaux au cours du développement embryonnaire (Figure $1 B$ ). Au jour 20, elles commencent à s'engager définitivement dans le lignage kératinocytaire et à exprimer la kératine 14 (Figure 1B). Ce n'est toutefois qu'après 40 jours de traitement par BMP4 et l'acide ascorbique que l'ensemble des marqueurs caractéristiques des kératino- 
A

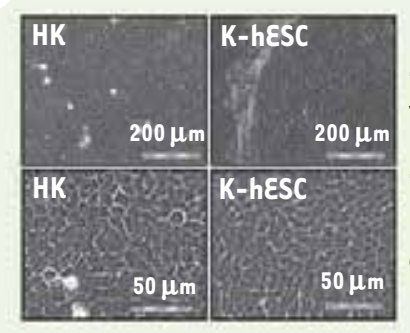

B 10

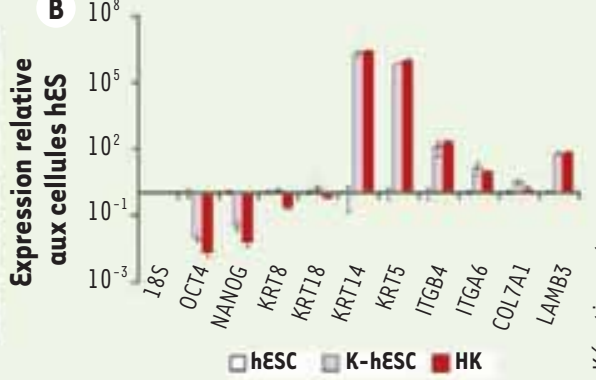

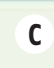

Kératine 5

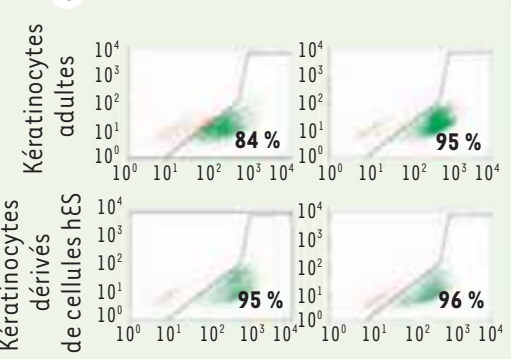

Figure 2. Établissement d'une population pure et homogène de kératinocytes dérivés de CSEh. Une fois le processus de différenciation terminé, des cellules présentant un phénotype similaire à celui des kératinocytes en culture peuvent être isolées et caractérisées. L’analyse par microscopie optique (à deux grossissements) montre que les kératinocytes dérivés des cellules souches embryonnaires (K-hESC) présentent une morphologie similaire à celle des kératinocytes adultes en culture ( $A$ ), ainsi qu'un même profil d'expression de marqueurs (KRT : kératine, COL : collagène, LAM : laminine) (B) et une immunopositivité pour les kératines 5 et 14 (C).

cytes est identifié sur une population de cellules qui a pu être isolée et amplifiée. Ces cellules appelés $\mathrm{K}$-hES pour «keratinocytes-derived human embryonic stem cells» présentent une morphologie en tout point similaire à celle des kératinocytes basaux adultes (Figure 2A). Ces cellules n'expriment plus OCT4 et NANOG, les facteurs de transcription caractéristiques des CSEh ; en revanche, elles sont positives pour l'ensemble des marqueurs caractéristiques des kératinocytes, les kératines 5 et 14 ( $95 \%$ de cellules positives), les chaînes alpha6 et bêta4 des intégrines et synthétisent des protéines de la matrice extracellulaire, dont le collagène de type VII et la laminine de type 5 (Figure $2 B$ et $C$ ).

La capacité de ces cellules à construire un épiderme pluristratifié a été mise en évidence in vitro, en ensemençant les cellules sur une matrice artificielle de polycarbonate ensuite placée à l'interface air/liquide pendant 10 jours. Dans ces conditions, les cellules poursuivent leur différenciation et forment un épiderme pluristratifié où l'on distingue l'ensemble des couches de l'épiderme humain (Figure 3A). Nous avons ensuite réalisé des études de greffe in vivo chez la souris (Figure 3B), préalable indispensable à une approche de thérapie cellulaire. Des épidermes dérivés in vitro de CSEh ont été greffés sur la région dorsale de souris immunodéficientes. Douze semaines après, le greffon présentait une architecture pluristratifiée similaire à celle d'une peau humaine adulte.

\section{Une recherche fondamentale applicable à la clinique}

Des CSEh différenciées en kératinocytes peuvent donc être utilisées comme source cellulaire pour la production d'un épiderme humain, expérimentalement du moins. Pour passer de cette preuve de concept à la clinique, nous avons bien sûr de nombreux défis à relever. Le premier est celui du changement d'échelle dont nous avons déjà eu l'occasion de présenter les différentes facettes dans ces colonnes [7]. Le second est celui de la sécurité du geste, qui se décline au moins selon deux axes, la tolérance immunitaire et le risque tumoral. $\varepsilon$ n ce qui concerne ce dernier, il n'existe pas encore de réponse absolue mais la multiplication actuelle des demandes d'autorisation d'essais cliniques auprès des agences réglementaires démontre au moins que la question est désormais posée dans des termes qui n'excluent pas l'accès au patient. Pour ce qui est de la réponse immunitaire, l'espoir est grand de voir des cellules d'origine embryonnaire provoquer des réponses très limitées, ne serait-ce que parce que des cellules fotales, un peu plus âgées, n'en ont pas elles-mêmes provoqué de massives [8].

Récemment, un nouveau type de cellules pluripotentes a vu le jour en offrant des perspectives quasi illimitées à la communauté scientifique internationale, les cellules appelées cellules souches « induites à la pluripotence (iPS)», cellules adultes reprogrammées présentant les mêmes caractéristiques de pluripotence et d'autorenouvellement que les CSEh. La création de centres de ressources biologiques inventoriant des kératinocytes dérivés d'iPS caractérisés et dont le phénotype CMH a été défini permettrait aux médecins de venir puiser, en fonction du besoin, des cellules présentant une combinaison de marqueurs du CMH compatible avec celle du malade en attente de greffe [9]. Nous proposerons d'utiliser ces lignées afin de produire des cellules de l'épiderme pour une thérapie cellulaire appliquée au traitement des grands brûlés mais également à d'autres pathologies cutanées telles que les génodermatoses ou les ulcérations qui, par exemple, sont une des complications chez un grand nombre de patients diabétiques. $\Delta$

Pluristratified epidermis

from human embryonic stem cells 


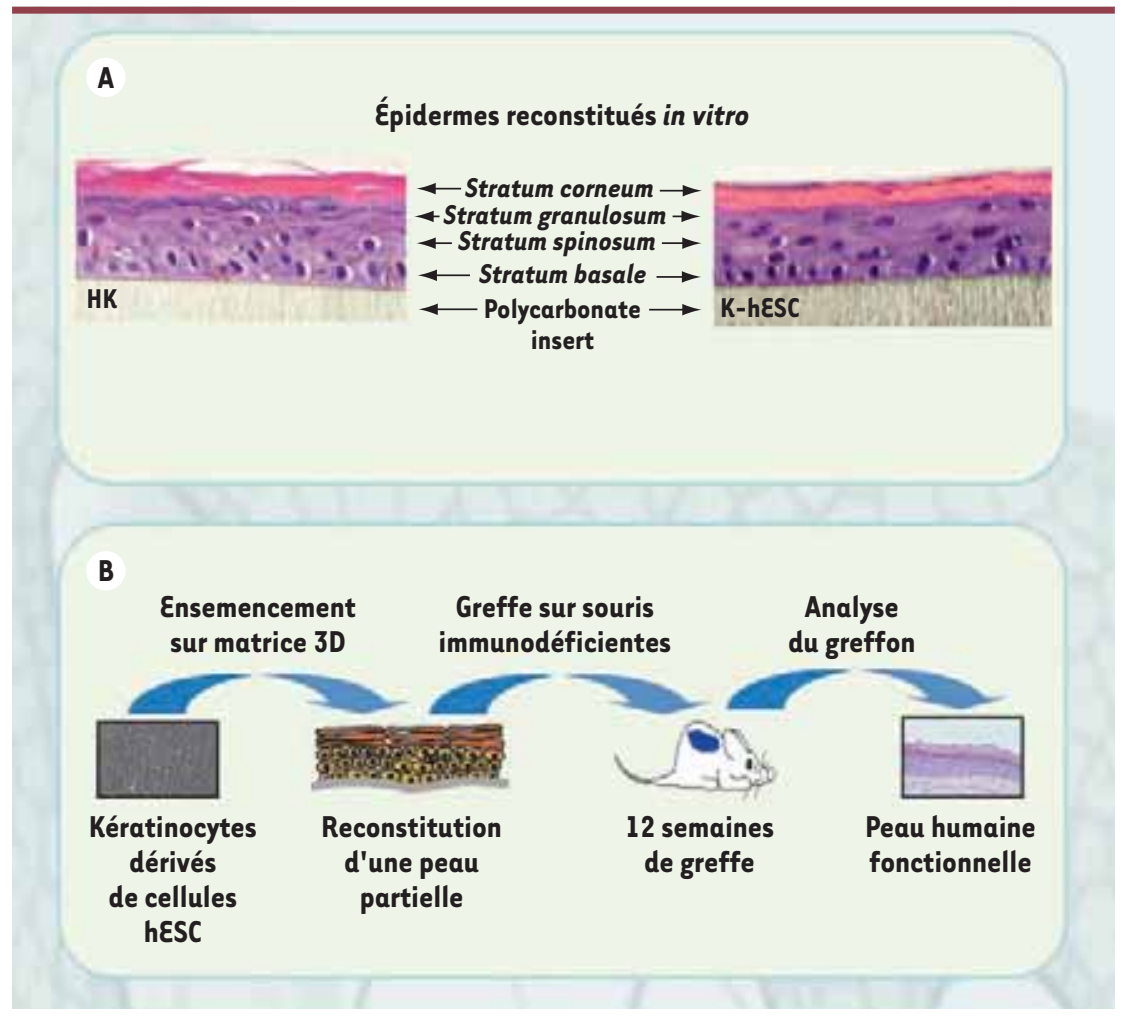

Figure 3. Construction d'un épiderme pluristratifié fonctionnel à partir de kératinocytes dérivés de CSEh in vitro et in vivo. Des kératinocytes adultes (HK) et des K-hESC ont ainsi été ensemencés en parallèle sur des matrices de polycarbonate. Après une première phase d'expansion de $48 \mathrm{~h}$ les cellules ont été placées à l'interface air/liquide afin d'induire leur stratification (A). Deux semaines plus tard, la coloration hématoxyline-éosine montre que les cellules K-hES sont capables de stratifier de la même façon que des kératinocytes adultes pour former l'ensemble des couches de l'épiderme (A). Des épidermes construits in vitro à partir de K-hESC ont été greffés chez des souris immunodéficientes $(B)$ après avoir été ensemencés sur une matrice tridimensionnelle. Quatre mois plus tard, l'analyse de la xénogreffe confirme la présence d'une peau artificielle humaine fonctionnelle.

\section{REMERCIEMENTS}

Ce travail a été soutenu par l'Institut national de la santé et de la recherche médicale (INSERM), l'Université Évry Val d'Essone (UદVE), l'Association française contre les myopathies (AFM), la Fondation René Touraine et Genopole.

\section{CONFLIT D'INTÉRÊTS}

Les auteurs déclarent n'avoir aucun conflit d'intérêts concernant les données publiées dans cet article.

\section{RÉFÉRENCES}

1. Gallico GG, O'Connor NE, Compton CC, et al. Permanent coverage of large burn wounds with autologous cultured human epithelium. $N$ Engl J Med $1984 ; 311: 448-51$

2. Metcalfe $A D$, Ferguson MW. Bioengineering skin using mechanisms of regeneration and repair. Biomaterials 2007 ; 28 : 5100-13.

3. Taylor CJ, Bolton EM, Pocock S, et al. Banking on human embryonic stem cells: estimating the number of donor cell lines needed for HLA matching. Lancet $2005 ; 366: 2019-25$.

4. Guenou $H$, Nissan X, Larcher F, et al. Human embryonic stem-cell derivatives for full reconstruction of the pluristratified epidermis: a preclinical study. Lancet $2009 ; 374$ : 1745-53.
5. Aubry L, Peschanski M, Perrier AL. Les cellules souches embryonnaires humaines pour la thérapie cellulaire de la maladie de Huntington. Med Sci (Paris) $2009 ; 25: 333-5$.

6. Rheinwald JG, Green H. Serial cultivation of human epidermal keratinocytes: the formation of keratinizing colonies from single cells. Cell 1975 ; $6: 331-4$.

7. Peschanski M. Cellules souches: I'heure venue du changement d'échelle. Med Sci (Paris) 2008 $24: 335-8$.

8. Touraine JL. Greffes de cellules souches et perspectives. Revue Médicale Suisse 2000 ; n 701 (online).

9. Nakatsuji N, Nakajima F, Tokunaga K. HLAhaplotype banking and iPS cells. Nat Biotechnol $2008 ; 26: 739-40$.

\section{Abonnez-vous}

à Médecine/Sciences
Bulletin d'abonnement page 110 dans ce numéro de $\mathrm{m} / \mathrm{s}$

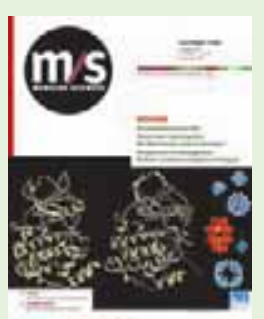

